\title{
PORTABLE TENT STRUCTURE DESIGNS FOR FLOOD PROTECTION
}

\author{
Róbert Nagy AND Zsolt GÁspár \\ Department of Structural Mechanics, Faculty of Civil Engineering \\ Budapest University of Technology and Economics \\ Müegyetem rkp. 3, 1111 Budapest, Hungary \\ robert.nagy@mail.bme.hu, gaspar@ep-mech.me.bme.hu
}

[Received: July 22, 2015, Accepted: September 30, 2015]

\begin{abstract}
Dedicated to Professor Barna Szabó on the occasion of his eightieth birthday and to Professor Imre Kozák on the occasion of his eighty-fifth birthday
\end{abstract}

\begin{abstract}
In this paper, we present two simple arrangements of portable tent structures for flood protection. In the first, an inflated cylinder lifts the top of the textile as the water level rises, while in the second the textile supporting the water pressure of the tide is hung on a steel framework and is filled with water previously. We give a numerical solution for the nonlinear system of equations of the compatibility and equilibrium conditions, and also present a complete analytic solution to the boundary value problem of the shape of an inextensible, weightless, prismatic textile, similar to the sessile drop problem. After discussing the mechanical behavior of the structures, different geometries capable of withstanding a certain water height are determined by a software developed for this specific purpose enabling the design for optimum. Furthermore, the basic rules of thumb are also formulated, aiding the preliminary conceptual design.
\end{abstract}

Mathematical Subject Classification: 65H05, 65B99

Keywords: Portable tent, flood protection, sessile drop, elastica, nonlinear pendulum, analytic solution

\section{INTRODUCTION}

Due to climate change, peak water levels more and more often exceed the height of the existing artificial embankment dams, causing ever increasing difficulties in flood-prone, inhabited areas near regulated rivers. Since the most common current emergency measures involve laying sandbags thus requiring considerable manpower, new methods are desired. In the first two chapters we present two possible alternatives, being fast and easy not only to transport but to construct as well, while in the last chapter we summarize the parametric representation of the directrix shape of a weightless, inextensible, prismatic membrane loaded by static water pressure, and we also derive the closed form equation of the area between the curve and an arbitrary vertical line. These formulations involve computationally burdensome elliptic 
functions and their inverses; therefore, solving directly the governing boundary value problem by a numerical algorithm is also a good alternative.

\section{First ARRANGEMENT}

The international Inflater project [1, managed by the MFKK Invention and Research Center and funded by the EU $7^{\text {th }}$ Framework Programme, aimed to develop novel, affordable mobile flood defense systems. One of the many possibilities presented there involves a self-erecting textile. As participants, we developed a computer program capable of determining the static and geometric properties of the structure 2]. The detailed description of the algorithm and the analysis of the structural behavior are given in this section.

2.1. Geometry. This portable structure, mounted on the vertical crown of the existing embankment dam, solely consists of tensile elements. The first component is an inflated cylinder of radius $r$ with diaphragms dividing its length into several cells and an additional active monitoring system for over-pressure control. The second is a sheet of impermeable textile skirt attached to a generatrix of the cylinder. The third component involves several tie-backs of length $l$ welded to the same generatrix at equal distances along the length of the dam. The latter two are anchored to the foundation, a common fixed point on the horizontal crown of the embankment dam. A representative equilibrium cross-section is depicted in Fig. 1. We describe the setup in the $\mathrm{x}-\mathrm{y}$ coordinate system. The main input parameters of the structure geometry are the BCDA textile section length $\left(l_{\mathrm{BCDA}}\right)$, the $\mathrm{AB}$ tie-back length $\left(l_{\mathrm{AB}}\right)$, the inflated cylinder radius $(r)$, and the supported water level above crown level $(h)$.

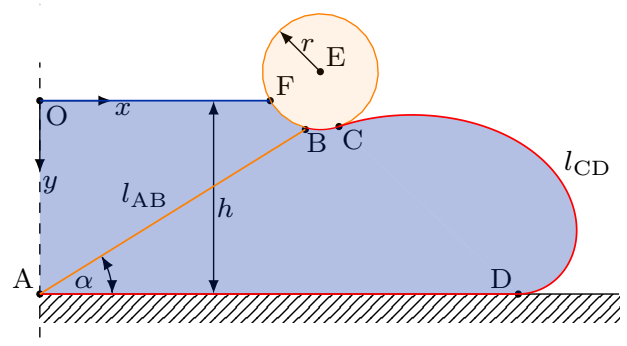

Figure 1. Cross-section of the $1^{\text {st }}$ arrangement

2.2. Behavior of the structure. Initially the structure lies on the ground and is erected as the floating cylinder filled with air lifts the top of the textile with the rising water level, while the tie-back - capable of letting the water through - prevents the whole structure from collapsing. To avoid leakage, diaphragms and an electronic monitoring system are applied. The sufficiency of the anchorage at point A, although a critical issue of the general stability, is not addressed here. 


\subsection{Assumptions.}

- The cross-section of the structure shows only negligible alterations in the longitudinal direction based on three-dimensional finite element simulation results, therefore it is sufficient to examine only the cross section in planar deformation state after linearly distributing the concentrated tie-back forces along directrix B.

- The over-pressure inside the inflated cylinder is high enough to maintain the circular cross-section, thus we handle it as a rigid body.

- The dimensions $l_{\mathrm{BCDA}}, l_{\mathrm{AB}}$, and $r$ are constant, that is, all elements have tensile stiffness large enough for the loads to cause negligibly small strains.

- The weight of the textile, the tie-back, and the cylinder are negligible compared to the loads they bear.

2.4. Theoretical background. The internal forces and the equilibrium geometry of the arrangement are determined by a system of four nonlinear transcendental equations. The first two express the equilibrium of the cylinder, while the other two state the geometric compatibility of the textile.

2.4.1. Loads on the cylinder. Fig. 2 shows the loads acting on the inflated cylinder. These are:

1. $T$, the specific tensile force from the tie-back,

2. $S$, the specific force from the textile tangential to the cylinder, and

3. $p(\xi)$ the pressure from the water normal to the cylinder.

We describe the conformation of the whole arrangement by four parameters:

1. $\alpha$, the inclination of the tie-back from the horizontal,

2. $\beta$, the angle between the horizontal and the BE line connecting the end of the tie-back and the center of the cylinder,

3. $\delta$, the angle between the $\mathrm{BE}$ line and the $\mathrm{CE}$ line connecting the point where the textile separates from the cylinder and the center point, and

4. $T$, the specific force in the tie-back.

In terms of these parameters the coordinates of the center of the cylinder (E) are

$$
x_{\mathrm{E}}=l_{\mathrm{AB}} \cos \alpha+r \cos \beta, \quad y_{\mathrm{E}}=h-l_{\mathrm{AB}} \sin \alpha-r \sin \beta,
$$

the coordinates of the point where the canvas separates from the cylinder $(\mathrm{C})$ are

$$
x_{\mathrm{C}}=x_{\mathrm{E}}-r \cos (\beta+\delta), \quad y_{\mathrm{C}}=y_{\mathrm{E}}+r \sin (\beta+\delta),
$$

the inclination of the tangent of the textile from the horizontal at point $\mathrm{C}$ is

$$
\varphi_{\mathrm{C}}=\frac{\pi}{2}-\beta-\delta
$$

the inclination of the FE line connecting the point where the water level intersects the cylinder and the center of the cylinder from the horizontal is

$$
\gamma=-\arcsin \frac{y_{\mathrm{E}}}{r},
$$




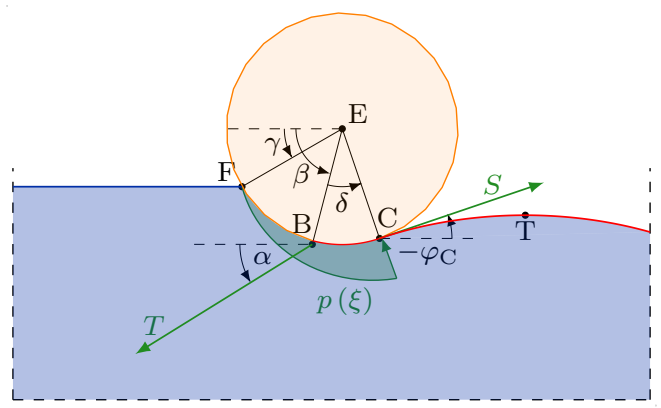

Figure 2. Parameters for the load calculation of the inflated cylinder

and the water pressure on the cylinder expressed as a function of the polar angle $(\xi)$ measured counterclockwise from the horizontal is

$$
p(\xi)=\varrho g r(\sin \xi-\sin \gamma),
$$

where $\rho=1000 \mathrm{~kg} / \mathrm{m}^{3}$ is the density of the water and $g=9.81 \mathrm{~m} / \mathrm{s}^{2}$ is the gravitational acceleration.

2.4.2. Equilibrium of the cylinder. The specific tensile force in the textile yields from the moment equilibrium at point $\mathrm{E}$ :

$$
S=T \sin (\beta-\alpha) .
$$

The two projections of the force equilibrium equation are:

$$
\begin{gathered}
\int_{\gamma}^{\beta+\delta} p(\xi) r \cos \xi \mathrm{d} \xi-T \cos \alpha+S \cos \varphi_{\mathrm{C}}=0, \\
-\int_{\gamma}^{\beta+\delta} p(\xi) r \sin \xi \mathrm{d} \xi+T \sin \alpha-S \sin \varphi_{\mathrm{C}}=0,
\end{gathered}
$$

which after integration and substitution of 2.3 and 2.6 lead to the first two transcendental condition equations:

$$
\begin{aligned}
f_{1}(\alpha, \beta, \delta, T) & =T[\sin (\beta-\alpha) \sin (\beta+\delta)-\cos \alpha]+ \\
& +\varrho g r^{2}\left[\frac{\cos ^{2} \gamma+3 \sin ^{2} \gamma-\cos 2(\beta+\delta)}{4}-\sin \gamma \sin (\beta+\delta)\right]=0, \\
f_{2}(\alpha, \beta, \delta, T) & =T[\sin (\beta-\alpha) \cos (\beta+\delta)+\sin \alpha]+ \\
& +\varrho g r^{2}\left[\frac{\gamma-\beta-\delta}{2}+\frac{\sin 2(\beta+\delta)+\sin 2 \gamma}{4}+\sin \gamma \cos (\beta+\delta)\right]=0,
\end{aligned}
$$

where $\gamma$ is determined via 2.4 and 2.1. 
2.4.3. Geometric compatibility of the textile. The remaining two condition equations:

$$
\begin{aligned}
& f_{3}(\alpha, \beta, \delta, T)=l_{\mathrm{BCDA}}-\delta \cdot r-l_{\mathrm{CD}}-x_{\mathrm{D}}=0, \\
& f_{4}(\alpha, \beta, \delta, T)=h-y_{\mathrm{D}}=0,
\end{aligned}
$$

where $l_{\mathrm{CD}}, x_{\mathrm{D}}$, and $y_{\mathrm{D}}$ are calculated through 4.15 , 4.20 , and $(4.9)_{2}$, respectively, stem from the compatibility conditions of the textile, whose loaded shape determination is described in Section 4.

2.4.4. The nonlinear system of equations. To summarize (2.8) and 2.9), we write:

$$
\mathbf{f}(\mathbf{x})=0, \quad \text { where } \quad \mathbf{f}=\left[\begin{array}{l}
f_{1} \\
f_{2} \\
f_{3} \\
f_{4}
\end{array}\right], \quad \text { and } \quad \mathbf{x}=\left[\begin{array}{c}
\alpha \\
\beta \\
\delta \\
T
\end{array}\right]
$$

We solve 2.10 by a target code applying the Jacobi method implemented in MATLAB 2011a (The MathWorks Inc., Natick, Massachusetts, United States). As stated in (4.7), to find a physically admissible solution that prevents the textile from rising above water level, that is, to prevent inflection in the directrix, the domain of $\mathbf{f}$ is restricted so that the Eötvös number exceeds the critical value of 1 .

\subsection{Analysis of the structure.}

2.5.1. Water level elevation. As an illustration, the equilibrium geometries of the arrangement $l_{\mathrm{AB}}=1.1 \mathrm{~m}, l_{\mathrm{BCDA}}=3.1 \mathrm{~m}, r=0.2 \mathrm{~m}$ are shown in the frames of Fig. 3 for water level rising by 10 centimeters. The corresponding specific tensile force increases in the tie-back $(T)$ and in the textile $(S)$, along with the tie-back inclination $(\alpha)$, are shown in Fig. 4

2.5.2. Maximal supported water level. The maximal supported water level $h$ is a function of $l_{\mathrm{AB}}, l_{\mathrm{BCDA}}$, and $r$ together describing the geometry of a given arrangement. This extremal state is defined by $\gamma=-\pi / 2$. We first consider the value of $r$ as given and instead of $\delta$ we choose $\varphi_{\mathrm{C}}$ as the independent variable, constraining $y_{\mathrm{C}}=r\left(1+\cos \varphi_{\mathrm{C}}\right)$. To reach the desired $h=y_{\mathrm{D}}$, which due to 4.7$)_{1}$ and 4.9$)_{2}$ is smaller than

$$
h_{\max }=\sqrt{\frac{2 r^{2}\left(1+\cos \varphi_{\mathrm{C}}\right)^{2}}{1-\cos \varphi_{\mathrm{C}}}},
$$

$S$ is found from $(4.9)_{2}$ to be

$$
S=\frac{\rho g\left(h^{2}-y_{\mathrm{C}}^{2}\right)}{2\left(1+\cos \varphi_{\mathrm{C}}\right)} .
$$

$\varphi_{\mathrm{C}}$ uniquely describes $F_{x}$ and $F_{y}$, the components of the water pressure resultant as shown in Fig. 5 and in 2.13:

$$
F_{x}=\frac{\varrho g r^{2}}{2}\left(1+\cos \varphi_{\mathrm{C}}\right)^{2}, \quad F_{y}=\frac{\varrho g r^{2}}{2}\left[\varphi_{\mathrm{C}}-\pi+\sin \varphi_{\mathrm{C}}\left(2+\cos \varphi_{\mathrm{C}}\right)\right] .
$$

Through the equilibrium of the cylinder, the tie-back position is determined via 

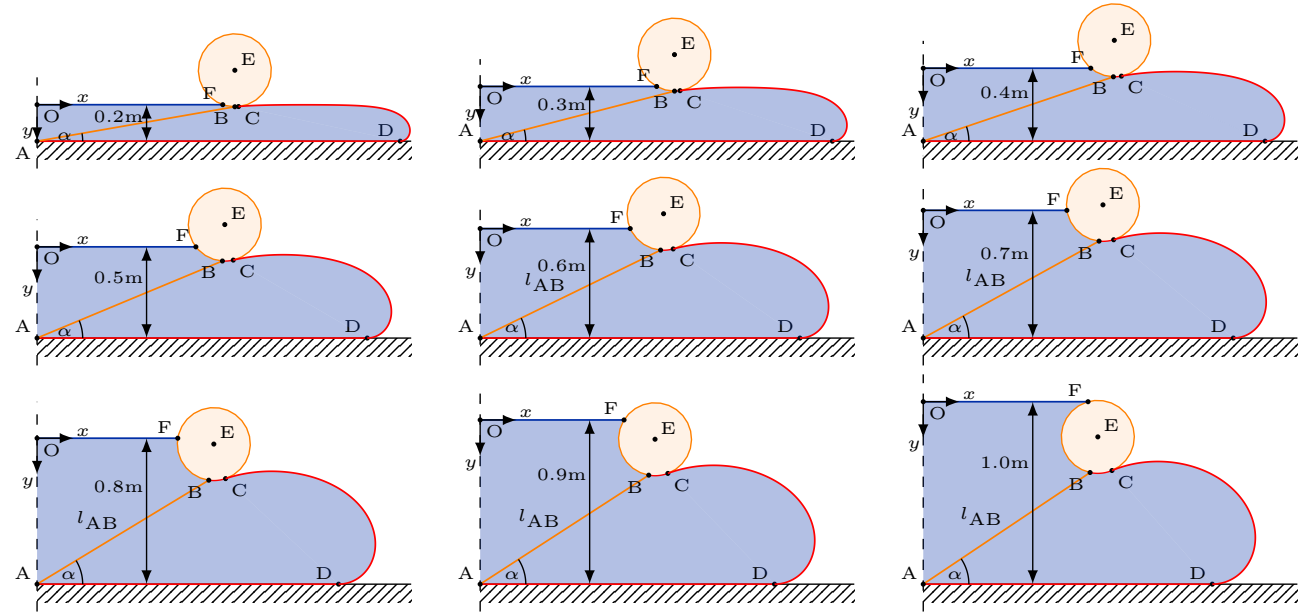

Figure 3. Frames of the equilibrium geomery of the arrangement $l_{\mathrm{AB}}=1.1 \mathrm{~m}, l_{\mathrm{BCDA}}=3.1 \mathrm{~m}, r=0.2 \mathrm{~m}$ in case of the water level rising by $10 \mathrm{~cm}$ in each step.
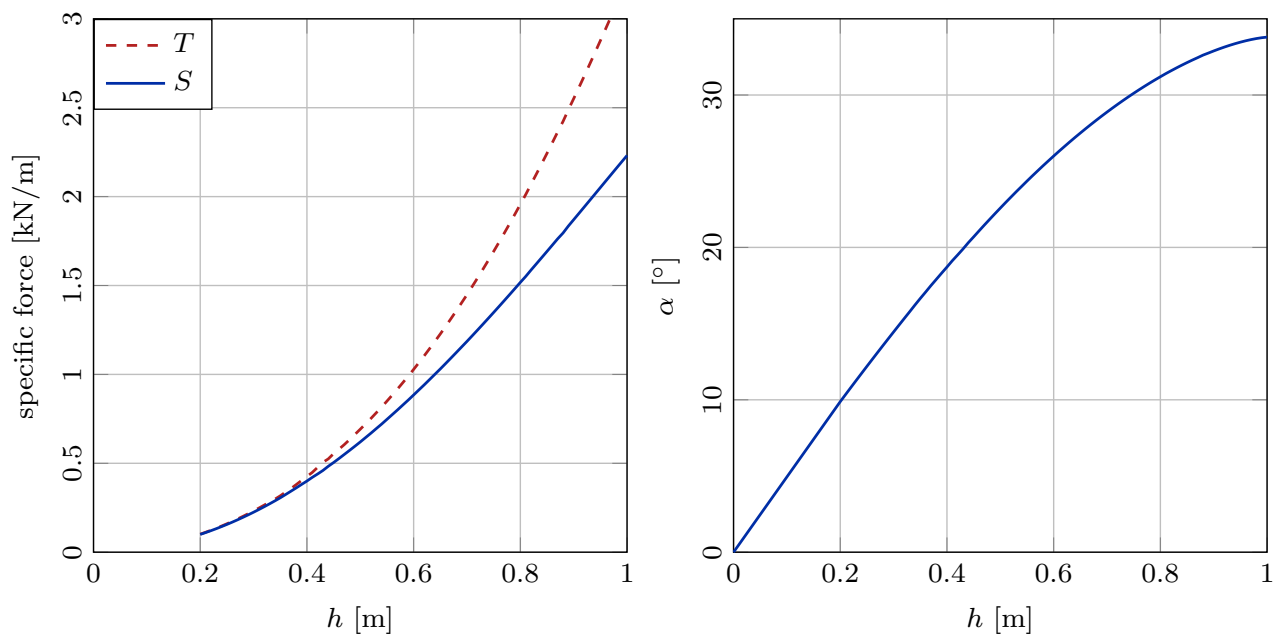

Figure 4. Specific tensile forces in the tie-back $(T)$ and in the textile $(S)$ (left), and the tie-back inclination $(\alpha)$ as a function of the rising water level $(h)$

$$
\alpha=\arctan \frac{-S \sin \varphi_{\mathrm{C}}-F y}{S \cos \varphi_{\mathrm{C}}+F x}, \quad \beta=\alpha+\arcsin \frac{F_{y} \cos \alpha+F_{x} \sin \alpha}{F_{y} \cos \varphi_{\mathrm{C}}-F_{x} \sin \varphi_{\mathrm{C}}},
$$




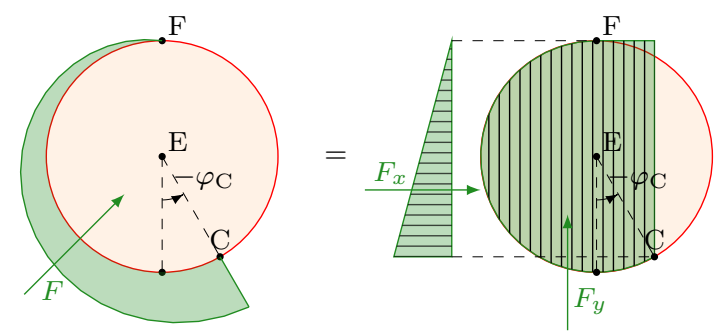

Figure 5. Decomposition of the water pressure resultant exerted on the cylinder in case of the highest possible supported water level

yielding the tie-back and textile lengths:

$$
l_{\mathrm{AB}}=\frac{h-r(1+\sin \beta)}{\sin \alpha}, \quad l_{\mathrm{BCDA}}=\delta \cdot r+l_{\mathrm{CD}}+x_{\mathrm{D}}
$$

where $\delta, l_{\mathrm{CD}}$ and $x_{\mathrm{D}}$ are calculated via 2.3 , 4.15 and 4.20, respectively.

\subsection{Preliminary design.}

We have now the possible geometric parameters characterizing the extremal design state of the structure. For a given $r$, their relation describes a surface in the threedimensional $\left(l_{\mathrm{AB}}, l_{\mathrm{BCDA}}, h\right)$ space, the contours of which are shown in Fig. 6 by blue lines, where it becomes possible to optimize the cost of the arrangement. Usually the textile length is to be minimized, both for financial reasons, and because the top of the existing dam it is going to be placed on offers a limited space, consequently the optimal designs are extremal points of the level lines forming the red curve also shown in Fig. 6. Agreeing upon this condition, we present the design method to define a geometry which is capable of withstanding a water height $h$ :

1. We assume that $h$ includes the uncertainty of the water level and the surface wave amplitudes as well. Hence reducing the problem to a quasi-static investigation.

2. As Fig. 7 shows, the tie-back and textile lengths decrease radically with the increasing cylinder radius, the critical design parameter, chosen to be the highest feasible value.

3. $l_{\mathrm{AB}}$ and $l_{\mathrm{BCDA}}$ are concluded form Fig. 7 .

4. $S$ and $T$, the specific textile and tie-back forces are found from Fig. 8

5. For designing the anchorage foundation, the tie-back inclination angle is shown in Fig. 9 


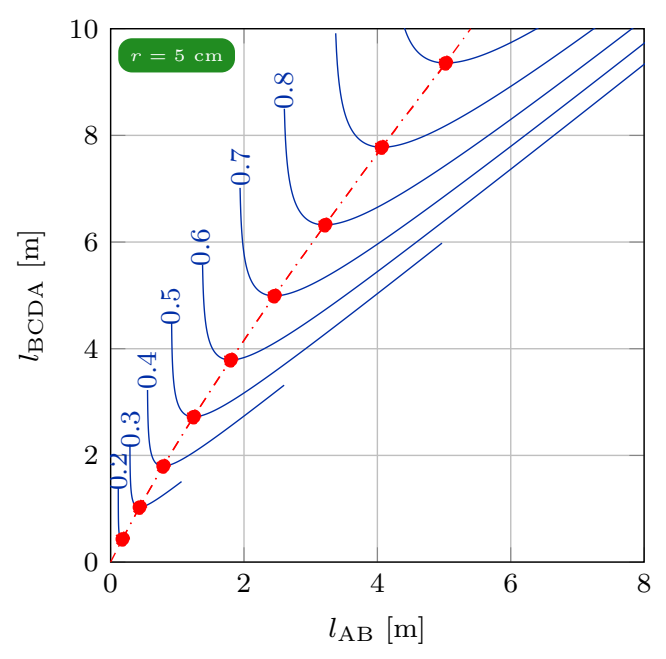

Figure 6. Blue level lines of the maximal supported water level, $h$ [m] for a given cylinder radius $(r=5 \mathrm{~cm})$; red curve is the optimal choice of the geometric parameters.
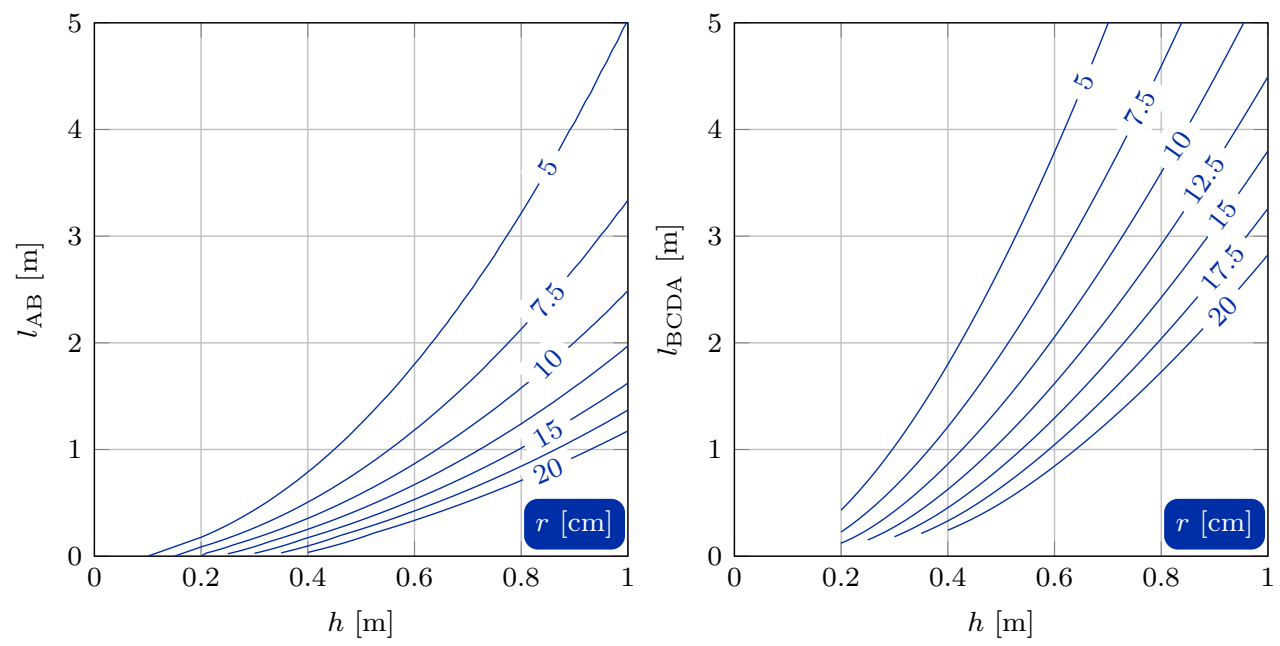

Figure 7. Tie-back and textile lengths as functions of the cylinder radius and maximal supported water level
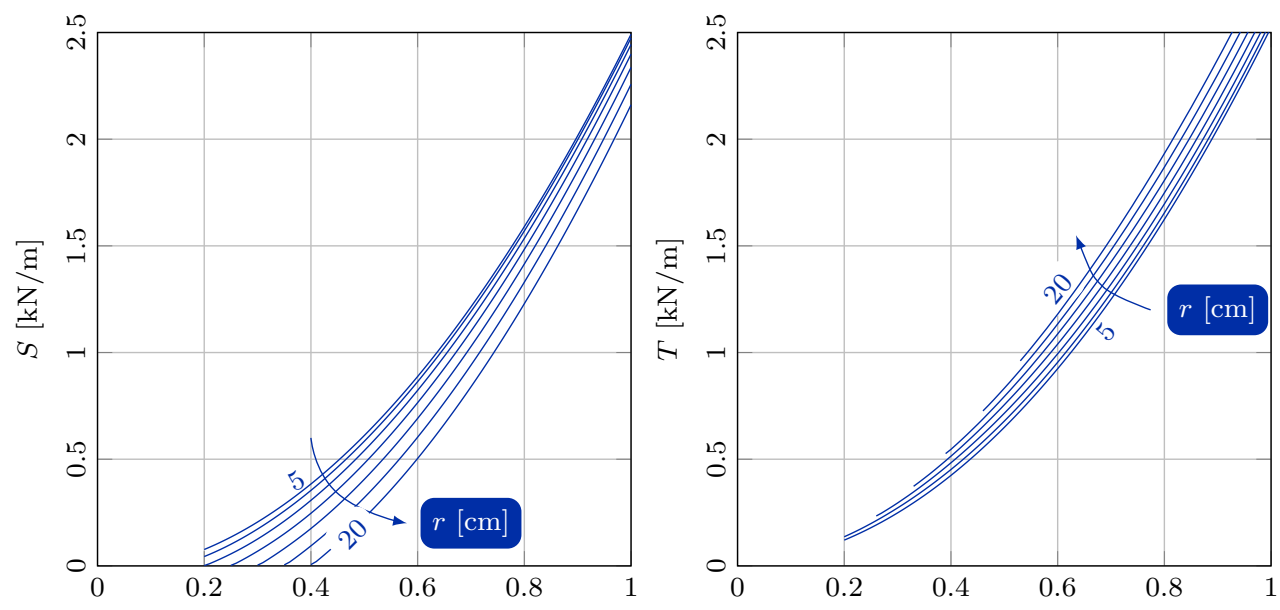


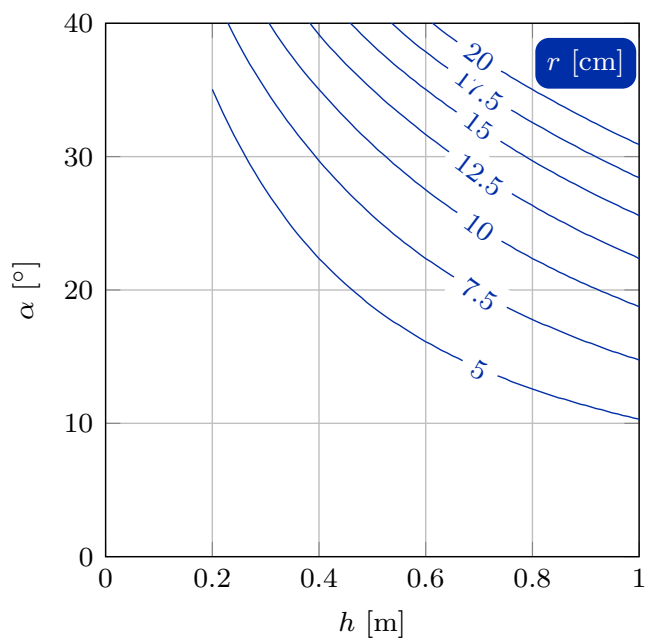

Figure 9. Extremal tie-back inclination angle as functions of the cylinder radius and maximal supported water level

\section{SECOND ARRANGEMENT}

The second scenario is invoked to life by the Airdome-Mobildam Kft. who approached us to analyze the structure presented in this chapter. Similarly to the first problem, we give here the shape and the specific forces of the textile and the reaction forces on the supports.

3.1. Geometry. This arrangement, also mounted on the crown, consists of an impermeable textile channel attached to a steel framework. A representative loaded cross-section solely of the textile in the plane of a frame position is presented in Fig. 10. The textile of cross-sectional length $L$ is suspended on the framework at height $H$ along two fixed directors, $\mathrm{B}_{1}$ and $\mathrm{B}_{2}$ at a vertical distance $d$ from each other. The inner water height is $h_{\mathrm{i}}$, the supported tidal water height over the crown is $h_{\mathrm{s}}$.

3.2. Behavior of the structure. Initially the unloaded textile is hanging on the supporting framework at points $\mathrm{B}_{1}$ and $\mathrm{B}_{2}$. During the fill-up, water is pumped inside the textile from the rising tide of the river, gradually erecting the yet symmetric structure. The rising supported water level deforms the structure violating the symmetry. At this stage, the weight of the water infill acts as a gravity retaining wall. 


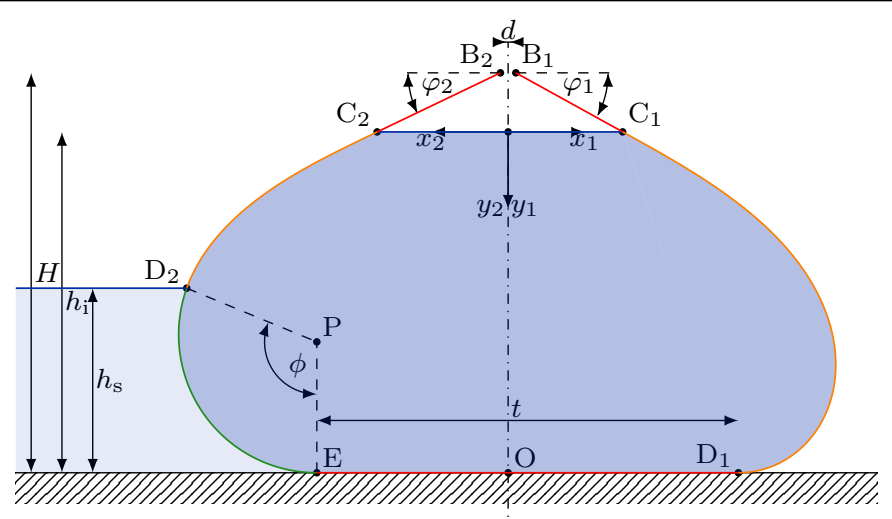

Figure 10. Cross-section of the $2^{\text {nd }}$ arrangement

\subsection{Assumptions.}

- The fixed suspension points $\left(\mathrm{B}_{1}\right.$ and $\left.\mathrm{B}_{2}\right)$ are connected in the axial direction with sufficiently stiff elements for the textile to have translational symmetry in that direction. This way the textile is in a planar deformation state.

- The weight of the textile is negligible compared to the perpendicular water pressure it bears, which itself is considered as a static loading. Consequently, the specific tensile forces $S_{1}$ in the $\mathrm{B}_{1} \mathrm{C}_{1} \mathrm{D}_{1}$ and $S_{2}$ in the $\mathrm{B}_{2} \mathrm{C}_{2} \mathrm{ED}_{2}$ textile sections are constant but not necessarily equal.

- All the elements have tensile stiffnesses large enough for the loads to cause negligibly small strains.

3.4. Unloaded, erected textile shape. Without the tidal water load the erected structure is symmetric, therefore we only present the right half $\left(\mathrm{B}_{1} \mathrm{C}_{1} \mathrm{D}_{1}\right)$ of the arrangement $H=1.6 \mathrm{~m}, d=5 \mathrm{~cm}$ and $L=6 \mathrm{~m}$ in Fig. 11 with internal water level rising by 20 centimeters. The whole textile is smooth, stretches straight in segment $\mathrm{B}_{1} \mathrm{C}_{1}$, leans against the ground in $\mathrm{D}_{1} \mathrm{O}$ and takes the loaded shape defined in Chapter 4 in segment $\mathrm{C}_{1} \mathrm{D}_{1}$. Note, that the horizontal dimension of the structure remains virtually unaltered during the process, thus the approximate formula for the preliminary design to calculate the horizontal space occupied is:

$$
t_{\mathrm{a}}=\frac{L^{2}-4 H^{2}-d^{2}}{2(L-d)} .
$$

3.4.1. Governing equation. The textile shape results from Chapter 4 , while the geometric compatibility of the structure as a whole for a given $h_{\mathrm{i}}$ internal water level is fulfilled by adjusting $S_{1}$ in

$$
f_{1}\left(S_{1}\right)=2\left(\frac{H-h_{\mathrm{i}}}{\sin \varphi_{1}}+l_{\mathrm{C}_{1} \mathrm{D}_{1}}+x_{\mathrm{D}_{1}}\right)-L=0,
$$




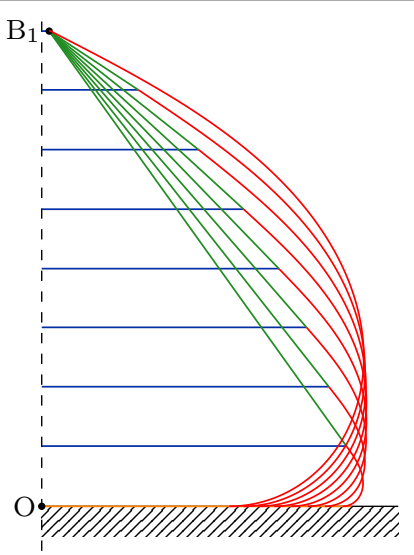

Figure 11. Unloaded, erected textile shape during the fillup process with internal water level rising by 20 centimeters

where $l_{\mathrm{C}_{1} \mathrm{D}_{1}}$ and $x_{\mathrm{D}_{1}}$ are found from 4.15 and 4.20 , respectively. Based on the restriction on the Hamiltonian of the system $\left(H_{0}<0\right.$ see Section 4.2 , or - in a mechanically more expressive form - the horizontal equilibrium of a textile section, the viable values of $S_{1}$ are taken from $\left(S_{1, \min }, \infty\right)$, where

$$
S_{1, \min }=\frac{\varrho g h_{\mathrm{i}}^{2}}{4} \text {. }
$$

We obtain the solution of $(3.2)$ by the bisection method, consequently this unbounded interval is transformed to the bounded interval of $s_{1} \in(0,1]$ by introducing

$$
s_{1}=\frac{S_{1, \min }}{S_{1}} .
$$

3.4.2. Design parameters. For calculating the necessary strength and stability of the frame the support force components are to be determined. In case of our example we illustrate them in Fig. 12 in terms of the internal water level elevation. 

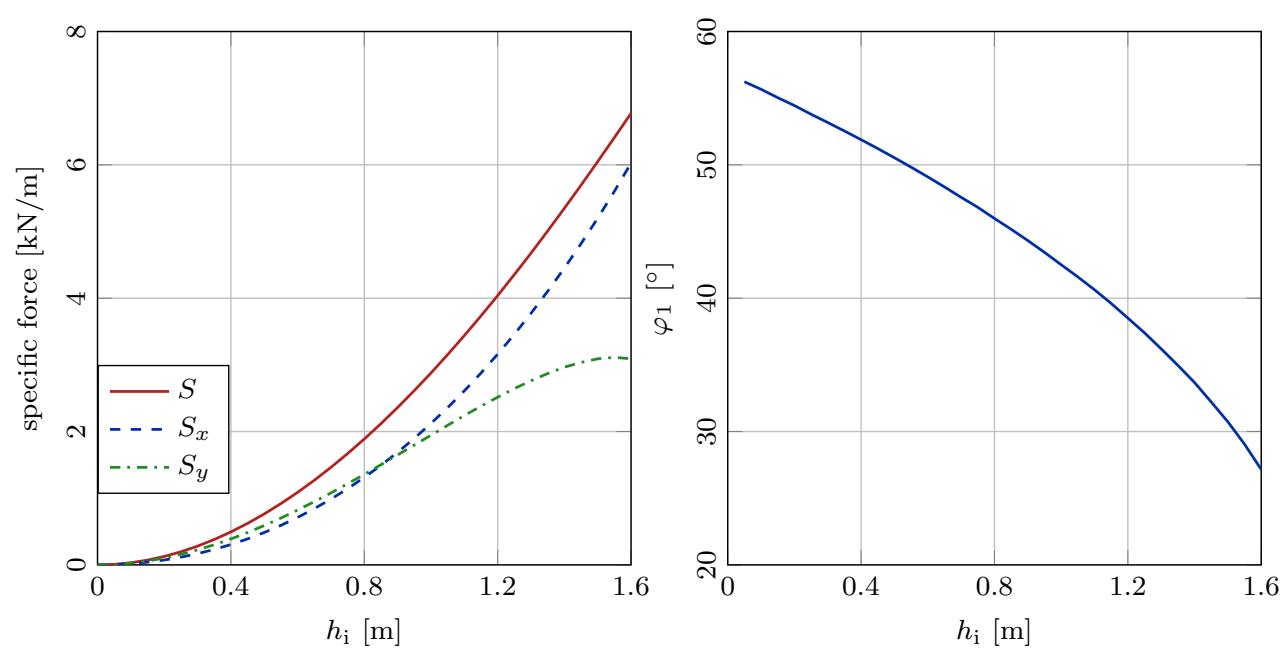

Figure 12. Specific tensile forces and its components in the textile (left), and initial inclination of the textile (right) as functions of the rising internal water level

3.5. Loaded geometry. The textile still remains smooth, stretches straight in segment $\mathrm{B}_{2} \mathrm{C}_{2}$, leans against the ground in $\mathrm{ED}_{1}$, takes the loaded shape defined in Chapter 4 in segment $\mathrm{C}_{2} \mathrm{D}_{2}$, and forms a circle section in $\mathrm{D}_{2} \mathrm{E}$, the radius $(R)$ and central angle $(\phi)$ of which is:

$$
\begin{gathered}
R=\frac{S_{2}}{\varrho g\left(h_{\mathrm{i}}-h_{\mathrm{s}}\right)}, \\
\phi=\arccos \frac{R-h_{\mathrm{s}}}{R} .
\end{gathered}
$$

3.5.1. Governing equation. The geometric compatibility for a given $h_{\mathrm{i}}$ and $h_{\mathrm{s}}$ is again met by adjusting $S_{2}$ in the cross-sectional length error:

$$
f_{2}\left(S_{1}\right)=2\left(\frac{H-h_{\mathrm{i}}}{\sin \varphi_{2}}+l_{\mathrm{C}_{2} \mathrm{D}_{2}}+R \phi+x_{\mathrm{D}_{2}}-R \sin \phi\right)-L=0,
$$

where $l_{\mathrm{C}_{2} \mathrm{D}_{2}}$ and $x_{\mathrm{D}_{2}}$ are found by evaluating at $\varphi=\pi-\phi$ the functions 4.13 and (4.19), respectively. Similarly to the unloaded case, the solution is obtained by the bisection method, thus the unbounded interval of $S_{2} \in\left(S_{2, \min }, \infty\right)$, where

$$
S_{2, \min }=\frac{\varrho g\left(h_{\mathrm{i}}^{2}-h_{\mathrm{s}}^{2}\right)}{4}
$$

is transformed to the bounded interval of $s_{2} \in(0,1]$ by introducing

$$
s_{2}=\frac{S_{2, \min }}{S_{2}} .
$$


3.6. Rise of the supported water level. Once the desired internal water level is reached, its volume (found from (4.21) and shown in Fig. 13) does not change throughout the increasing loading of the tide. Numerically, after the two internal

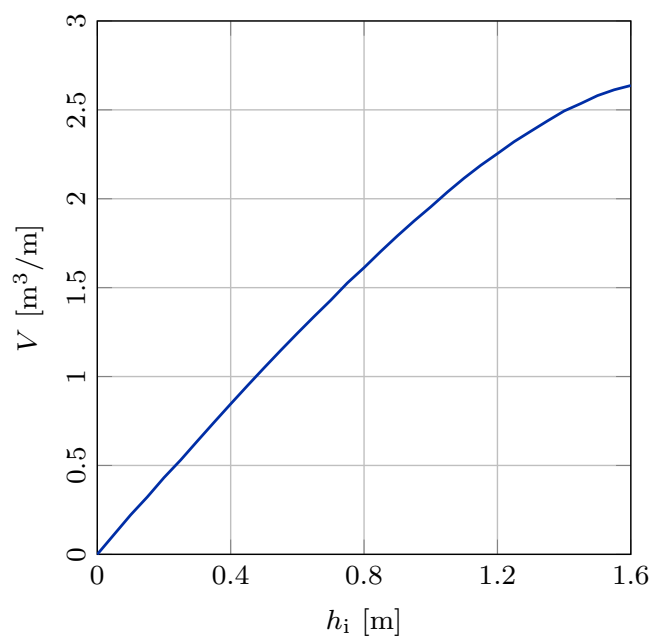

Figure 13. Specific volume of the infill against its rising level

iteration of $S_{1}$ and $S_{2}$ for the geometric compatibility of the two sides, an outer bisection iteration adjusts $h_{\mathrm{i}}$ to match the volume requirement:

$$
\begin{aligned}
f_{3}\left(h_{\mathrm{i}}\right) & =2 V\left(\varphi_{\mathrm{C}}, \pi, S\right)-V\left(\varphi_{\mathrm{C}_{1}}, \pi, S_{1}\right)+V\left(\varphi_{\mathrm{C}_{2}}, \varphi_{\mathrm{D}_{2}}, S_{2}\right)+ \\
& +\frac{R^{2} \phi}{2}-\frac{R^{2} \sin 2 \phi}{4}+R(1-\cos \phi)\left(x_{\mathrm{D}_{2}}-R \sin \phi\right)=0,
\end{aligned}
$$

where $V$ is defined in 4.21, arguments with indice 1 and 2 represent the free and the loaded side, respectively while the symmetric unloaded geometry is referred to by subscripts without any index. Fixing the infill volume at the value when $h_{\mathrm{i}}=1.3 \mathrm{~m}$ in the unloaded state, the increasing $h_{\mathrm{s}}$ deforms the cross-section shown in the frames of Fig. 14. Fig. 15 depicts the tension $\left(S_{2}\right)$ in the loaded textile decreasing with $h_{\mathrm{s}}$.

\subsection{Conclusions.}

- The structure acts as a gravity retaining wall.

- The symmetry of the erected structure is violated by the supported tidal water pressure.

- Counter-intuitively, instead of pushing the structure away from the riverbed, the external water pulls it closer due to the uplift.

- The internal water level decreases, although insignificantly (by $5 \mathrm{~cm}$ in the presented example, see Figure 15, where the specific tensile force becomes zero at $h_{\mathrm{s}}=1.25 \mathrm{~cm}$ ). 

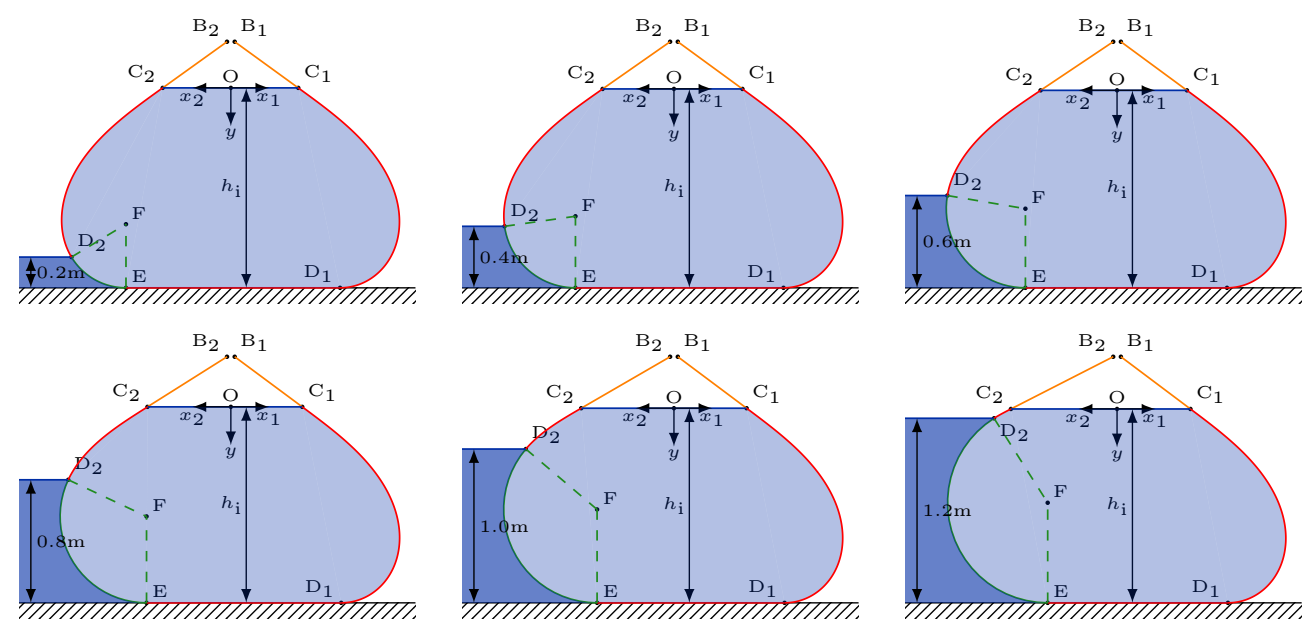

Figure 14. Frames of the structure deformation as the supported water level rises by 20 centimeters

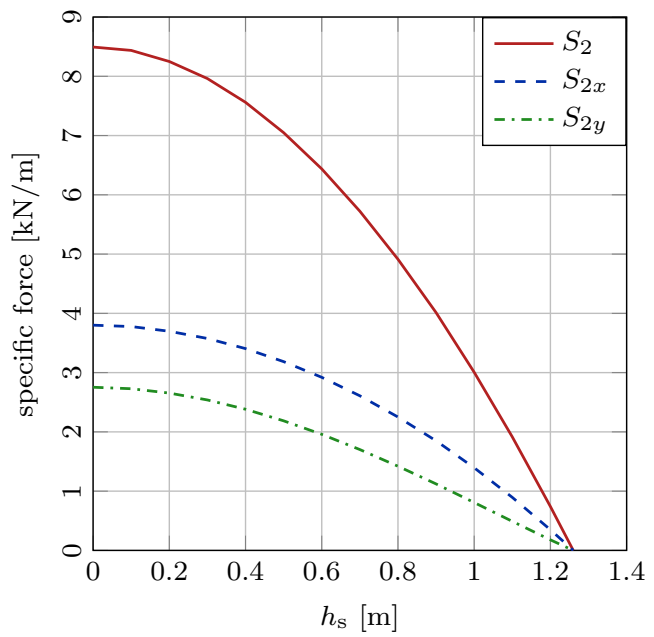

Figure 15. Specific tensile force and its components in the textile loaded by the rising supported water level

- The textile tension in the supporting side decreases to zero as $h_{\mathrm{s}}$ reaches $h_{\mathrm{i}}$, when the supporting textile loosens and its shape becomes undefined.

- The properties of the side away from the river depend only on the internal water level, which is virtually unaltered, thus its shape and tension agrees with the unloaded state. 
- $h_{\mathrm{s}}=h_{\mathrm{i}}$ is the critical state from stability point of view, when total load of the tide acts only on the outer textile part, while the balancing tension in the other side of the textile is missing.

3.8. Preliminary design. The preliminary design for an expected $h_{\mathrm{s}, \max }$ starts with prescribing $H$ and $d$ for the framework and $t$, the necessary minimal length where the textile touches the ground at the time of the maximal tidal level to prevent the river flooding out and to provide the overall stability of the structure; and results in the critical design force descriptors from the point of view of the framework: $S_{1}$, the specific force in the outer textile part and $\varphi_{\mathrm{C}_{1}}$, the inclination of the textile tangent at point $\mathrm{C}_{1}$. In Figure 16 , we present the design charts supporting this simplified work-flow. We consider the limit case when $h_{\mathrm{i}}=1.25 h_{\mathrm{s}, \max }$, and assume that $H=h_{\mathrm{i}}$. Having been agreed upon the safety ratio of 1.25 and infill ratio of 1 , the designer chooses a suitable $t$, adjusts it by $d$ and from the left graph on Figure 16 the necessary cross-sectional textile length is obtained alongside with $S_{1}$ as is $\varphi_{\mathrm{C}_{1}}$ from the chart on the right. For a given $H$, the increase in $L$ causes a decrease in both $S_{1}$ and $\varphi_{\mathrm{C}_{1}}$, thus not only because of the limited space available and cost efficiency reasons, but because of overall structural stability issues, the shortest $L$ is to be chosen meeting the criterion on $t$.
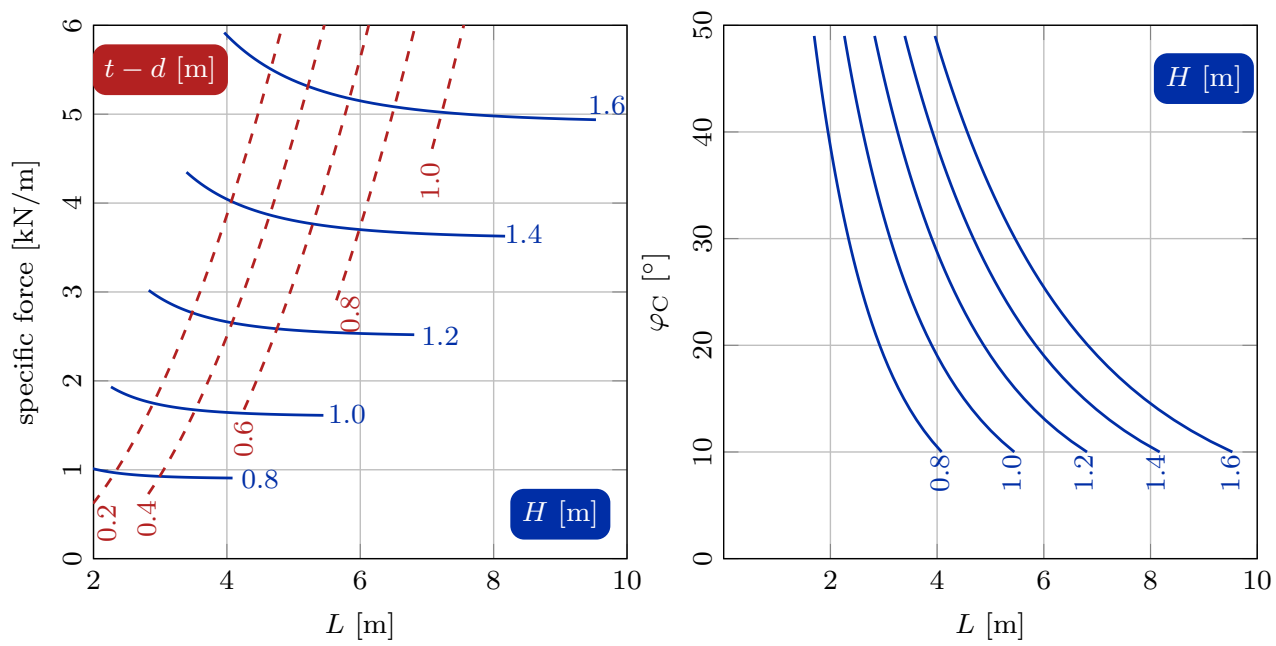

Figure 16. Dependence of the specific force in the outer textile part $\left(S_{1}\right)$ (left) and the inclination of the textile tangent from the vertical at point $\mathrm{C}_{1}\left(\varphi_{\mathrm{C}_{1}}\right)$ (right) on the cross-sectional textile length $(L)$ and the framework height $(H)$ when the supported water height $\left(h_{\mathrm{s}}\right)$ is $0.8 H$ 


\section{Shape of the LOADED TeXtile SECTION}

Consider a prismatic, weightless, inextensible textile supporting static water pressure, the cross-section of which is shown in Fig. 17, where the water level is at $y=0$. In this section we give the parametric functional description of this directrix (in the following referred to as fiber) shape and the closed form equation of the $V$ area between the curve and the $y$ axis. For similar structures, such as closed pressurized geomembrane tubes c.f. 3] for the elastic case, [4 for stacked arrangement, 5] for infill with liquids of different density, 6] for compressible subgrade soil; or 7 for liquid filled nonlinearly elastic shells.

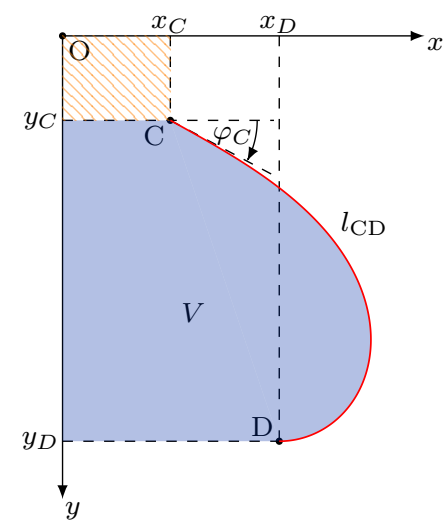

Figure 17. Boundary value problem of the textile shape loaded by static water pressure

4.1. Initial value problem. The initial value problem of ordinary differential equations describing the fiber shape is given by

$$
\begin{aligned}
x^{\prime} & =\cos \varphi, \quad x(0)=x_{C}, \\
y^{\prime} & =\sin \varphi, \quad y(0)=y_{C}, \\
\varphi^{\prime} & =\frac{\varrho g}{S} y, \quad \varphi(0)=\varphi_{C},
\end{aligned}
$$

where $x$ and $y$ are the horizontal and vertical coordinates respectively, $\varphi$ is the inclination of the tangent from the positive $x$ axis in clockwise direction. $\varrho$ is the water density, $g$ is the gravitational acceleration, $S$ is the specific tensile force in the fiber, and prime denotes the derivative with respect to the arch length parameter $(\lambda)$. The first two equations are the compatibility conditions and the third is the tangential equilibrium statement. Since the fiber weight is negligible and the water pressure is normal to the curve, the equilibrium condition in the normal direction prescribes the specific tension $(S)$ in the fiber being constant. By eliminating $y$ from the last two 
ODEs of 4.1), we simplify the problem to

$$
\varphi^{\prime \prime}=\omega^{2} \sin \varphi, \quad \varphi^{\prime}(0)=\omega^{2} y_{C}, \quad \varphi(0)=y_{C}, \quad \omega^{2}=\frac{\varrho g}{S},
$$

which is analogous to the nonlinear pendulum equation 8 except the sign change. The same equation governs the elastica [9] and capillarity problems and if we consider $S$ to be the surface tension, we arrive at the problem of sessile drops.

4.2. The first integral. We can define the corresponding Lagrangian:

$$
L=\varphi^{2}+4 \omega^{2} \sin ^{2} \frac{\varphi}{2}
$$

which does not depend explicitly on the arch-length, thus following Noether's theorem [10] we construct a conserved quantity:

$$
H=\varphi^{\prime 2}-4 \omega^{2} \sin ^{2} \frac{\varphi}{2}=\text { const. }
$$

This - speaking in mechanical terms - states the horizontal equilibrium of a textile section. At a certain depth the first term is proportional to the horizontal hydrostatic load resultant, the second is proportional to the horizontal tensile force in the textile, resulting in the alternate form of 4.4 :

$$
\tilde{H}=\frac{\rho g y^{2}}{2}+S \cos \varphi=\text { const. }
$$

In the followings we work with the form of 4.4). This translational symmetry, analogous to the energy or the Hamiltonian of the pendulum moving in conservative field, suggests scrutinizing the periodic phase space and its two qualitatively different trajectories DTCD $(0<H=$ const. $)$ and SUVS $(0>H=$ const. $)$ separated by the dash-dotted separatrix $(H=0)$ in Fig. 18 . We introduce two constants:

$$
H_{0}=\omega^{4} y_{C}^{2}-H_{p} \sin ^{2} \frac{\varphi_{C}}{2}, \quad H_{p}=4 \omega^{2},
$$

which correspond to the total energy and the maximal potential energy of the pendulum, respectively.

In case of the first arrangement (Chapter 2), we allow only the curves above the upper separatrix since it restricts the $y$ coordinate to be positive, that is the fibre does not rise above the water level, and has no inflection, which is the only physically acceptable scenario, analogous to the overturning pendulum. The necessary criterion is $H_{0}>0$, which yields an inequality constraint to the boundary conditions given by

$$
\begin{aligned}
& y_{C}>y_{l}, \quad \text { where } y_{l}=\sqrt{\frac{2\left(1-\cos \varphi_{c}\right) S}{\varrho g}}, \quad \text { or } \\
& E_{o}>1, \text { where } \quad E_{o}=\frac{\varrho g y_{c}^{2}}{2\left(1-\cos \varphi_{c}\right) S} .
\end{aligned}
$$

Here $y_{l}$ is the capillarity length and Eo is the Eötvös number both measuring the relationship between the gravity and surface tension effects, and are extensively used 


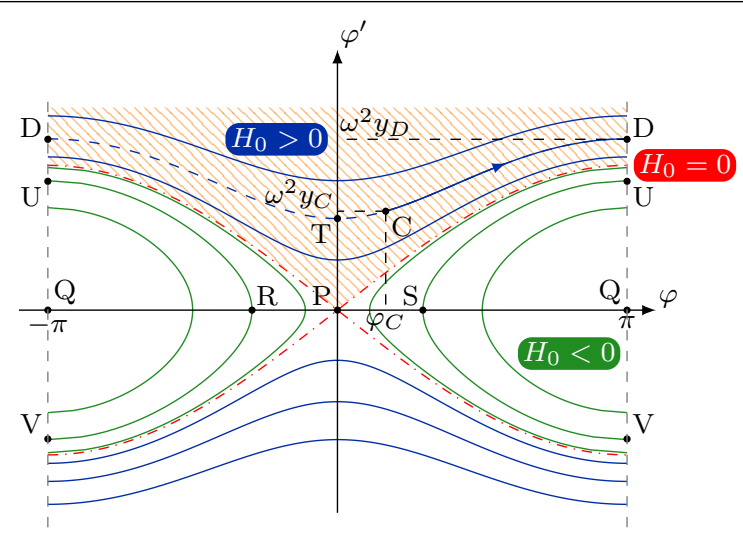

Figure 18. Phase space of the Hamiltonian

for analyzing capillarity phenomena such as the shape of pendant or sessile drops [1]. Now we are able to determine

$$
y(\varphi)=\sqrt{y_{C}^{2}+\frac{4}{\omega^{2}}\left(\sin ^{2} \frac{\varphi}{2}-\sin ^{2} \frac{\varphi_{C}}{2}\right)},
$$

which gives us the maximal and minimal textile heights denoted by points $\mathrm{D}$ and $\mathrm{T}$ in the phase space:

$$
\begin{aligned}
& y_{T}=\sqrt{y_{C}^{2}-\frac{2}{\omega^{2}}\left(1-\cos \varphi_{C}\right)}, \\
& y_{D}=\sqrt{y_{C}^{2}+\frac{2}{\omega^{2}}\left(1+\cos \varphi_{C}\right)} .
\end{aligned}
$$

In case of the second arrangement (Chapter 3), analogous to the oscillating pendulum, the only viable curves are between the separatrices $\left(H_{0}<0\right)$ with initial conditions $\varphi^{\prime}=0$ and $\varphi>0$, turning around the relations in 4.7, but leaving 4.8 unaltered.

4.3. Elliptic functions. In the followings we use the definition of Legendre's elliptic integrals 4.10 and the Jacobi functions 4.11. These are the incomplete elliptic integrals of the first (F) and second (E) kind; the Jacobi amplitude (am), the sinus amplitudinis (sn), and cosinus amplitudinis (cn) functions:

$$
\begin{gathered}
\mathrm{F}(\varphi \mid A)=\int_{0}^{\varphi} \frac{\mathrm{d} \phi}{\sqrt{1-A \sin ^{2} \phi}}, \\
\mathrm{E}(\varphi \mid A)=\int_{0}^{\varphi} \sqrt{1-A \sin ^{2} \phi} \mathrm{d} \phi \\
\operatorname{am}(\lambda \mid A)=\mathrm{F}^{-1}(\lambda \mid A), \\
\operatorname{sn}(\lambda \mid A)=\sin [\operatorname{am}(\lambda \mid A)], \\
\operatorname{cn}(\lambda \mid A)=\cos [\operatorname{am}(\lambda \mid A)]
\end{gathered}
$$


4.4. Parametric description of the fiber. In the first scenario $H_{p}=4 \omega^{2}>0$, $H_{0}>0$, and $\varphi^{\prime}>0$; in the second $H_{0}<-H_{p} \sin ^{2}\left(\varphi_{\mathrm{C}} / 2\right)<0$ and $\varphi^{\prime}>0$ since $\varphi \in\left[\varphi_{\mathrm{C}}, \pi\right]$. Consequently, for both arrangements 4.4 results in the ODE:

$$
\varphi^{\prime}=\sqrt{H_{0}+H_{p} \sin ^{2} \frac{\varphi}{2}}
$$

and its solutions:

$$
\begin{gathered}
\lambda(\varphi)=\operatorname{sign}\left(H_{0}\right) \frac{2}{\sqrt{H_{0}}} \mathrm{~F}\left(\frac{\varphi}{2} \mid A\right)-\lambda_{C}, \quad \text { and } \quad \varphi(\bar{\lambda})=2 \operatorname{am}(\bar{\lambda} \mid A), \quad \text { where } \\
\bar{\lambda}=\operatorname{sign}\left(H_{0}\right) \frac{\sqrt{H_{0}}}{2}\left(\lambda+\lambda_{C}\right), \quad \lambda_{C}=\operatorname{sign}\left(H_{0}\right) \frac{2}{\sqrt{H_{0}}} \mathrm{~F}\left(\frac{\varphi_{C}}{2} \mid A\right), \quad A=-\frac{H_{p}}{H_{0}} .
\end{gathered}
$$

Hence the length of the CD curve is

$$
l_{\mathrm{CD}}=\operatorname{sign}\left(H_{0}\right) \frac{2}{\sqrt{H_{0}}}\left[\mathrm{~F}\left(\frac{\varphi_{\mathrm{D}}}{2} \mid A\right)-\mathrm{F}\left(\frac{\varphi_{\mathrm{C}}}{2} \mid A\right)\right] .
$$

Substituting 4.13 2 into 4.8 gives

$$
y(\bar{\lambda})=\sqrt{y_{C}^{2}+\frac{4}{\omega^{2}}\left[\operatorname{sn}^{2}(\bar{\lambda} \mid A)-\sin ^{2} \frac{\varphi_{C}}{2}\right]} .
$$

Merging $4.2{ }_{1}$ and $4.132_{2}$, we get

$$
x^{\prime}=\cos [2 \operatorname{am}(\bar{\lambda} \mid A)]=1-2 \operatorname{sn}^{2}(\bar{\lambda} \mid A),
$$

and its solution

$$
\begin{aligned}
x(\bar{\lambda})=x_{\mathrm{C}} & +\frac{4 H_{0}+2 H_{p}}{\sqrt{H_{0}} H_{p}}\left(\bar{\lambda}-\bar{\lambda}_{\mathrm{C}}\right)+ \\
& +\frac{4 \sqrt{H_{0}}}{H_{p}}\left\{\mathrm{E}\left[\operatorname{am}\left(\bar{\lambda}_{\mathrm{C}} \mid A\right) \mid A\right]-\mathrm{E}[\operatorname{am}(\bar{\lambda} \mid A) \mid A]\right\},
\end{aligned}
$$

where $\bar{\lambda}_{\mathrm{C}}=\sqrt{H_{0}} \lambda_{\mathrm{C}} / 2$, or in terms of $\varphi$

$$
\begin{aligned}
x(\varphi)=x_{\mathrm{C}} & +\operatorname{sign}\left(H_{0}\right) \frac{4 H_{0}+2 H_{p}}{\sqrt{H_{0}} H_{p}}\left[\mathrm{~F}\left(\frac{\varphi}{2} \mid A\right)-\mathrm{F}\left(\frac{\varphi_{\mathrm{C}}}{2} \mid A\right)\right]+ \\
& +\operatorname{sign}\left(H_{0}\right) \frac{4 \sqrt{H_{0}}}{H_{p}}\left[\mathrm{E}\left(\frac{\varphi_{\mathrm{C}}}{2} \mid A\right)-\mathrm{E}\left(\frac{\varphi}{2} \mid A\right)\right] .
\end{aligned}
$$

We also give the $x$ coordinate of point $\mathrm{D}$ :

$$
x_{\mathrm{D}}=x_{\mathrm{C}}+l_{\mathrm{CD}} \frac{H_{p}+2 H_{0}}{H_{p}}+\operatorname{sign}\left(H_{0}\right) \frac{4 \sqrt{H_{0}}}{H_{p}}\left[\mathrm{E}\left(\frac{\varphi_{\mathrm{C}}}{2} \mid A\right)-\mathrm{E}\left(\frac{\varphi_{\mathrm{D}}}{2} \mid A\right)\right] .
$$


4.5. Enclosed area. The area between the $y$ axis and the curve, when $\varphi \in\left[\varphi_{\mathrm{C}}, \varphi_{\mathrm{D}}\right]$ (shaded area in Fig. 17), can be deduced using Green's theorem, partial integration, variable change, 4.16 and 4.1 1,3 in this order, as shown in 4.21.

$$
\begin{aligned}
V\left(\varphi_{\mathrm{C}}, \varphi_{\mathrm{D}}, S\right) & =\int_{y_{\mathrm{C}}}^{y_{\mathrm{D}}} x \mathrm{~d} y=\int_{0}^{l_{\mathrm{CD}}} x y^{\prime} \mathrm{d} \lambda=[x y]_{\mathrm{C}}^{\mathrm{D}}-\int_{0}^{l_{\mathrm{CD}}} x^{\prime} y \mathrm{~d} \lambda= \\
& =[x y]_{\mathrm{C}}^{\mathrm{D}}-\int_{\varphi_{\mathrm{C}}}^{\varphi_{\mathrm{D}}} \frac{x^{\prime} y}{\varphi^{\prime}} \mathrm{d} \varphi=x_{\mathrm{D}} y_{\mathrm{D}}-x_{\mathrm{C}} y_{\mathrm{C}}-\int_{\varphi_{\mathrm{C}}}^{\varphi_{\mathrm{D}}} \frac{\cos \varphi y}{\omega^{2} y} \mathrm{~d} \varphi= \\
& =x_{\mathrm{D}} y_{\mathrm{D}}-x_{\mathrm{C}} y_{\mathrm{C}}-\frac{1}{\omega^{2}} \int_{\varphi_{\mathrm{C}}}^{\varphi_{\mathrm{D}}} \cos \varphi \mathrm{d} \varphi= \\
& =x_{\mathrm{D}} y_{\mathrm{D}}-x_{\mathrm{C}} y_{\mathrm{C}}+\left(\sin \varphi_{\mathrm{C}}-\sin \varphi_{\mathrm{D}}\right) \frac{S}{\rho g}
\end{aligned}
$$

4.6. Conclusion. In this section we derived the parametric functional description of the cross-sectional shape and the explicit function of the enclosed area of an inextensible weightless prismatic textile subjected to water pressure loading and constant specific tensile force with known initial point coordinates $\left(x_{\mathrm{C}}, y_{\mathrm{C}}\right)$ and prescribed initial tangent line inclination $\left(\varphi_{\mathrm{C}}\right)$. As a summary, we give here the most important equations of the fiber position:

$$
\begin{aligned}
x(\varphi)=x_{\mathrm{C}} & +\operatorname{sign}\left(H_{0}\right) \frac{4 H_{0}+2 H_{p}}{\sqrt{H_{0}} H_{p}}\left[\mathrm{~F}\left(\frac{\varphi}{2} \mid A\right)-\mathrm{F}\left(\frac{\varphi_{\mathrm{C}}}{2} \mid A\right)\right]+ \\
& +\operatorname{sign}\left(H_{0}\right) \frac{4 \sqrt{H_{0}}}{H_{p}}\left[\mathrm{E}\left(\frac{\varphi_{\mathrm{C}}}{2} \mid A\right)-\mathrm{E}\left(\frac{\varphi}{2} \mid A\right)\right], \\
y(\varphi)= & \sqrt{y_{C}^{2}+\frac{4 S}{\rho g}\left(\sin ^{2} \frac{\varphi}{2}-\sin ^{2} \frac{\varphi_{C}}{2}\right)},
\end{aligned}
$$

and the area enclosed by the $\mathrm{y}$ axis and the curve:

$$
V(\varphi)=x y-x_{\mathrm{C}} y_{\mathrm{C}}+\left(\sin \varphi_{\mathrm{C}}-\sin \varphi\right) \frac{S}{\rho g},
$$

the parameter $(\varphi)$ being the inclination angle.

\section{REFERENCES}

1. Inflater project. 2015, URL http://inflater.eu/. [Online; accessed 19-March-2015].

2. Adany, S., Bocskai, Z., Galasko, G., Gaspar, Z., Lakatos, E., Lengyel, A., and NAGY, R.: INFLATER Handbook: Half time report, chap. Mechanical structure design, pp. 121-167. Springer Verlag, Budapest University of Technology and Economics, Geotechnical Department, l. nagy, t. huszak edn., 2013.

3. Antman, S. S. and Schagerl, M.: Slumping instabilities of elastic membranes holding liquids and gases. International Journal of Non-Linear Mechanics, 40(8), (2005), 11121138 .

4. Cantre, S.: Geotextile tubes - analytical design aspects. Geotextiles and Geomembranes, 20(5), (2002), 305-319. 
5. Malik, J. and Sysala, S.: Analysis of geosynthetic tubes filled with several liquids with different densities. Geotextiles and Geomembranes, 29(3), (2011), 249-256.

6. Guo, W., Chu, J., and Yan, S.: Effect of subgrade soil stiffness on the design of geosynthetic tube. Geotextiles and Geomembranes, 29(3), (2011), 277-284.

7. Kolesnikov, A.: Equilibrium of an elastic spherical shell filled with a heavy fluid under pressure. Journal of applied mechanics and technical physics, 51(5), (2010), 744-750.

8. Ochs, K.: A comprehensive analytical solution of the nonlinear pendulum. European Journal of Physics, 32(2), (2011), 479.

9. Levien, R.: The elastica: a mathematical history. Electrical Engineering and Computer Sciences University of California at Berkeley.

10. Noether, E.: Invariante variationsprobleme. Nachrichten von der Gesellschaft der Wissenschaften zu Göttingen, mathematisch-physikalische Klasse, 1918, (1918), 235-257.

11. de Gennes, P., Brochard-Wyart, F., and Quere, D.: Capillarity and Wetting Phenomena: Drops, Bubbles, Pearls, Waves. Springer, 2004, ISBN 9780387005928. 